\title{
Assessment of Pulmonary Artery Pulsatility by Multidetector Computed Tomography in Patients Affected by Chronic Obstructive Pulmonary Disease and Pulmonary Hypertension: Preliminary Data
}

\author{
Anna Grazia D’Agostino, ${ }^{1}$ Giuseppe Valerio, ${ }^{2}$ Pierluigi Bracciale, ${ }^{2}$ and Fabio Valerio ${ }^{2}$ \\ ${ }^{1}$ U. O. di Radiodiagnostica, Ospedale "A. Perrino", Via Appia 246, 72100 Brindisi, Italy \\ ${ }^{2}$ Divisione di Pneumologia "Antonio Blasi", Ospedale "Ninetto Melli", Via Lecce 246, 72017 San Pietro Vernotico, Italy
}

Correspondence should be addressed to Anna Grazia D’Agostino; pneumo@tiscali.it

Received 21 November 2012; Accepted 31 December 2012

Academic Editors: A. Celi and A. S. Melani

Copyright (C) 2013 Anna Grazia D’Agostino et al. This is an open access article distributed under the Creative Commons Attribution License, which permits unrestricted use, distribution, and reproduction in any medium, provided the original work is properly cited.

The aim was to assess if computed tomography is able to measure pulmonary artery pulsatility in patients affected by chronic obstructive pulmonary disease and to ascertain whether pulsatility is different in patients with and without pulmonary hypertension and whether it is related to haemodynamics. We selected two groups of patients, the first one with pulmonary hypertension and the second one without. In patient with hypertension, pulmonary artery pressure and resistance were increased with the increased diameters (transverse $36 \pm 5 \mathrm{~mm}$ and axial $38 \pm 4 \mathrm{~mm}$ versus $22 \pm 3$ and $25 \pm 5$, resp.), the increased cross-sectional area (10 \pm 08 versus $4 \pm 1 \mathrm{~cm}^{2}$ ), and the reduced pulsatility $(21 \pm 7$ versus $10 \% \pm 5 \%)$. Arterial stretching was decreased in patients with hypertension $(10 \pm 5$ versus $21 \% \pm 7 \%)$ and significantly related to pulmonary vascular resistances and pressure. Cardiac output measured by tomography was significantly related to that obtained by Fick method and was not different in the two groups. The diameters allow to identify patients with $\mathrm{PH}$, assuming a cut-off of $28 \mathrm{~mm}$ and assuming a pulsatility of right branch of $26 \%$ as well. These preliminary observations indicate tomography as a suitable technique, being able to measure the pulsatility and the dimensions of the arteries and the right ventricular functional parameters.

\section{Introduction}

The assessment of pulmonary hypertension $(\mathrm{PH})$ during the chronic obstructive pulmonary disease (COPD) is important, since $\mathrm{PH}$ worsens quality of life, prognosis, effort tolerance, and outcome in acute respiratory failure [1-3]. Precapillary $\mathrm{PH}$ can be seen in $30 \%$ to $43 \%$ of patients affected by COPD, reaching values higher than $45 \mathrm{mmHg}$ in $5 \%$ [1-3]. $\mathrm{PH}$ is determined by both functional vasoconstriction and vessels remodeling [3-5] that determine the right ventricular afterload leading to effort intolerance, poor quality of life, cardiac failure, and reduced life expectancy. The assessment of pulsatility (Puls) of pulmonary artery (PA) in PH looks like a promising tool, since it looks related to severity, progression of disease, functional capacity, prognosis, and survival [69]. The invasive right heart catheterization ( $\mathrm{RHC}$ ) is the gold standard for the diagnosis of $\mathrm{PH}$, and the assessment of reversibility of $\mathrm{PH}$ with vasodilators, the measure of Puls by intravascular ultrasound (IVU) [10-14]. The noninvasive cardiac echocardiography (US) allows the measurement of the pulmonary artery dimensions, the right ventriculare shape, the ejection fraction, and the noninvasive estimation of the systolic pulmonary artery pressure [15-18], but the applicability of US in patients affected by COPD is limited because of the reduced acoustic window [15], due to pulmonary hyperinflation. Within the imaging methods, magnetic resonance imaging (MRI) is extensively studied and allows the measurement of both morphologic and functional data such as Puls and pulmonary blood flow [19-21], but it is not widely available. Multidetector computer Tomography (MDCT) and cardiac software imaging are diffusely employed and available; they allow the detailed examination 
of heart, coronary vessels, left ventricular function, and pulmonary arteries diameters [22-28]. Our aims are to study whether MDCT allows the assessment of Puls in COPD, whether Puls is different between patients with and without $\mathrm{PH}$, and whether it is related to hypertension.

\section{Methods}

2.1. Selection of Patients. All the patients were affected by COPD (diagnosed according to ATS statement and GOLD criteria) [29], and they were examined as soon as they consecutively come in the ward of pulmonary division. Patients were assigned to a specific GOLD stage, according to post bronchodilator FEV1.

The inclusion criteria were (a) presence of COPD and (b) the written informed consent and motivation. The exclusion criteria were (a) the significant cardiac diseases such as valvulopathies or cardiac failure defined by a fraction ejection of left ventricle lesser than $50 \%$ (measured by echocardiography), (b) the presence of other pulmonary diseases as pulmonary fibrosis, tuberculosis, or chronic embolic disease (already diagnosed by scintigraphy), (c) the allergy to iodine compounds, (d) the recent significant radiation loads due to isotopic or radiological procedures, (e) the psychiatric disorders as claustrophobia or panic attacks, (f) the sleep apnoea syndrome (diagnosed by polysomnography), and (g) the absence of acute relapses. All the enrolled patients were examined by clinical check, pulmonary functional testing, blood gas analysis, echocardiography, six minutes walking test (6 MWD), RHC in the first day, and chest CT examination one day after. The study started on April 2008 and ended one year later. The operator was unaware of the presence of precapillary $\mathrm{PH}$ that was diagnosed under RHC by a mean pulmonary artery pressure higher than $25 \mathrm{mmHg}$ with a capillary pressure lower than $15 \mathrm{mmHg}$ measured during RHC. Within one year, only twenty patients with $\mathrm{PH}$ were recruited because of the low prevalence of the disease and the refuse of the informed consent; the enrolled patients were compared to the first consecutive twenty patients without $\mathrm{PH}$.

The goal to access the applicability of MDCT in the assessment of Puls was accomplished examining the success rate in the imaging of PA diameters and surface area in diastole and systole. The difference of Puls within patients with and without $\mathrm{PH}$ was obtained comparing the mean by a statistical package and observing the relationship between Puls and PAP or RVP.

2.2. Physiological Measurements. Lung volumes were measured by a whole body chest plethysmography (Pulmobox 6200, SensorMedics, California, USA). Measured volumes were referred to ERS normal standards [30]. Vital capacity (VC) and forced expiratory volume in the 1st second (FEV1) were reported.

Blood gas analysis was carried out by radial artery puncture (performed at rest whilst breathing air) and automated analysis (Rapidlab Bayer, FRG), measuring arterial oxygen tension $\left(\mathrm{PaO}_{2}\right)$ and haemoglobin oxygen saturation $\left(\mathrm{SaO}_{2}\right)$.
Mixed venous oxygen tension $\left(\mathrm{PvO}_{2}\right)$ and saturation $\left(\mathrm{SvO}_{2}\right)$ were obtained by pulmonary artery sampling.

Effort tolerance was measured by 6 MWD [31] and dyspnoea by WHO rating scale [32]. RHC was performed by cubital vein catheterization, according to Seldinger's technique using Swan-Ganz catheters (Baxter, USA) [33].

2.3. Haemodynamic Measurements. Pulmonary artery pressure (PAP) was assessed both in stable state and under NO $(20 \mathrm{ppm})$ inhalation. Cardiac output $\left(Q^{\prime}\right)$ was obtained using Fick method by means of simultaneous pulmonary and radial arteries sampling (to measure oxygen content) together with the measurement of oxygen consumption (Cortex Metalyzer, Germany). Pulmonary wedge pressure $(\mathrm{Pw})$ measurement allowed pulmonary vascular resistances (PVRs), computed dividing pressure drop by cardiac output $\left(\mathrm{PAP}-\mathrm{Pw} / \mathrm{Q}^{\prime}\right)$. All patients showed $\mathrm{Pw}$ lesser than $15 \mathrm{mmHg}$.

2.4. Imaging Measurements. Computed tomography was accomplished by a 64-detector tomograph (Philips Brilliance 64 , Germany). A first whole chest scanning was performed by a low dosage radiation without contrast medium with high resolution technique $(100 \mathrm{kV} ; 95 \mathrm{mAs} ; 64 \times 0,625 \mathrm{~mm}$; $t$ rot $0,75 \mathrm{sec}$; pitch 1.078) held at functional residual capacity and in forced expiration to check for concomitant pulmonary diseases or associated conditions. A "test bolus" technique was used before injecting the contrast medium (Iomeprol 400 , Bracco, Italy) to compute the time necessary for the simultaneous imaging of both the pulmonary trunk and the right ventricle, choosing as region of interest (ROI) the pulmonary trunk, the right atrium and the left ventricle. Afterwards the acquisition with contrast medium started, using cardiac gating $(120 \mathrm{kV} ; 350 \mathrm{mAs} ; 64 \times 0,625 \mathrm{~mm}$; $t$ rot 0,4 sec; pitch 0.299), from the aortic arch to the cardiac base, including the pulmonary arteries up to two centimetres from the thoracic cage wall in the reconstruction algorithm. The contrast medium was injected at a flow rate of $4 \mathrm{~mL} / \mathrm{sec}$ and at a concentration of $400 \mathrm{mg} / \mathrm{mL}$, by a double lumen injector, according to the sequence: $80 \mathrm{~mL}$ of solution with $60 \% \mathrm{mdc}$ and $40 \%$ sterile saline, followed by $40 \mathrm{~mL}$ of contrast medium and $40 \mathrm{~mL}$ of sterile saline. Two doctors measured the longitudinal and the transverse diameters of both pulmonary arteries in CT observed under mediastinal gating, choosing a plan perpendicular to the path of vessels. The measures were obtained at the end-systolic and the enddiastolic frames, carefully checking during acquisition that the cardiac frequency was in the range of 55 to $85 \mathrm{bpm}$. The end-systolic frame happens around the $40 \%$ of the cardiac cycle and the end-systolic frame around the 70\% (Figures 1 and 2). The cross-sectional area was measured as well, and the pulsatility of arteries was measured by the ratio between the systolic and the diastolic surface areas. By means of a dedicated software, it was possible to measure the ejection fraction and the cardiac output of right ventricle.

2.5. Statistical Analysis. Biometric data, pulmonary function, effort performance, and hemodynamic and radiologic variables of patients with and without pulmonary hypertension 


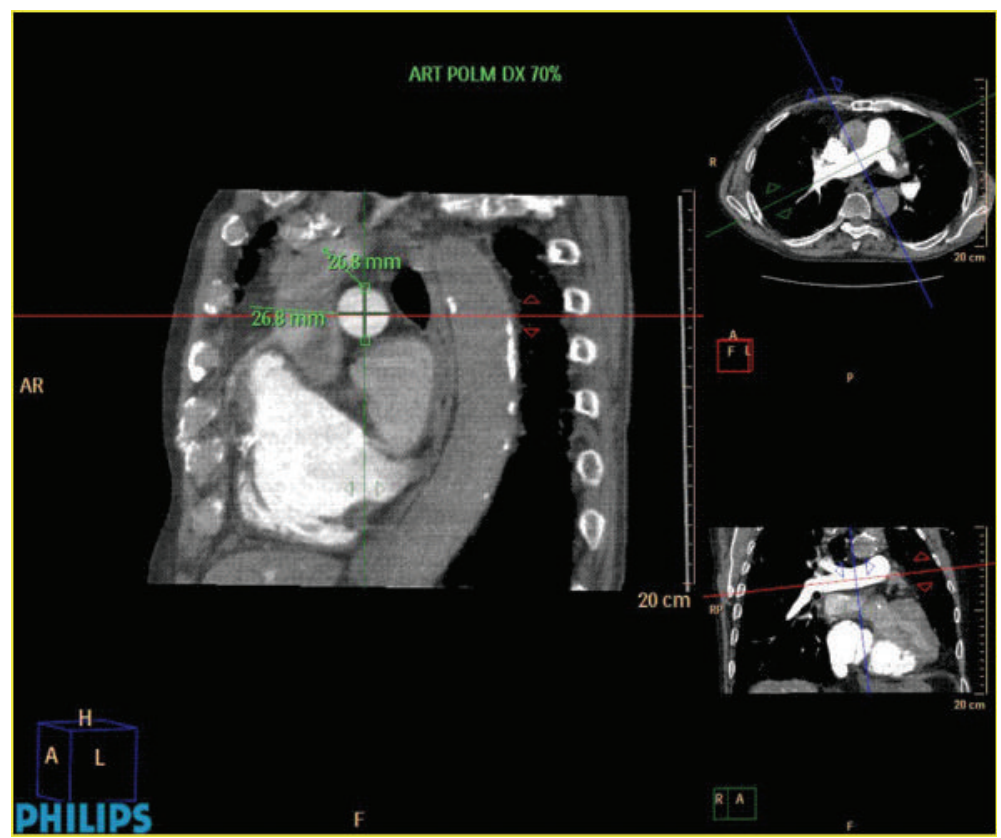

FIGURE 1: Assessment of pulmonary diameters in mediastinal window at end-diastolic phase. The measurement of pulmonary artery diameters was repeated in end-systolic and end-diastolic phases; the ratio allowed the assessment of pulsatility of pulmonary artery.

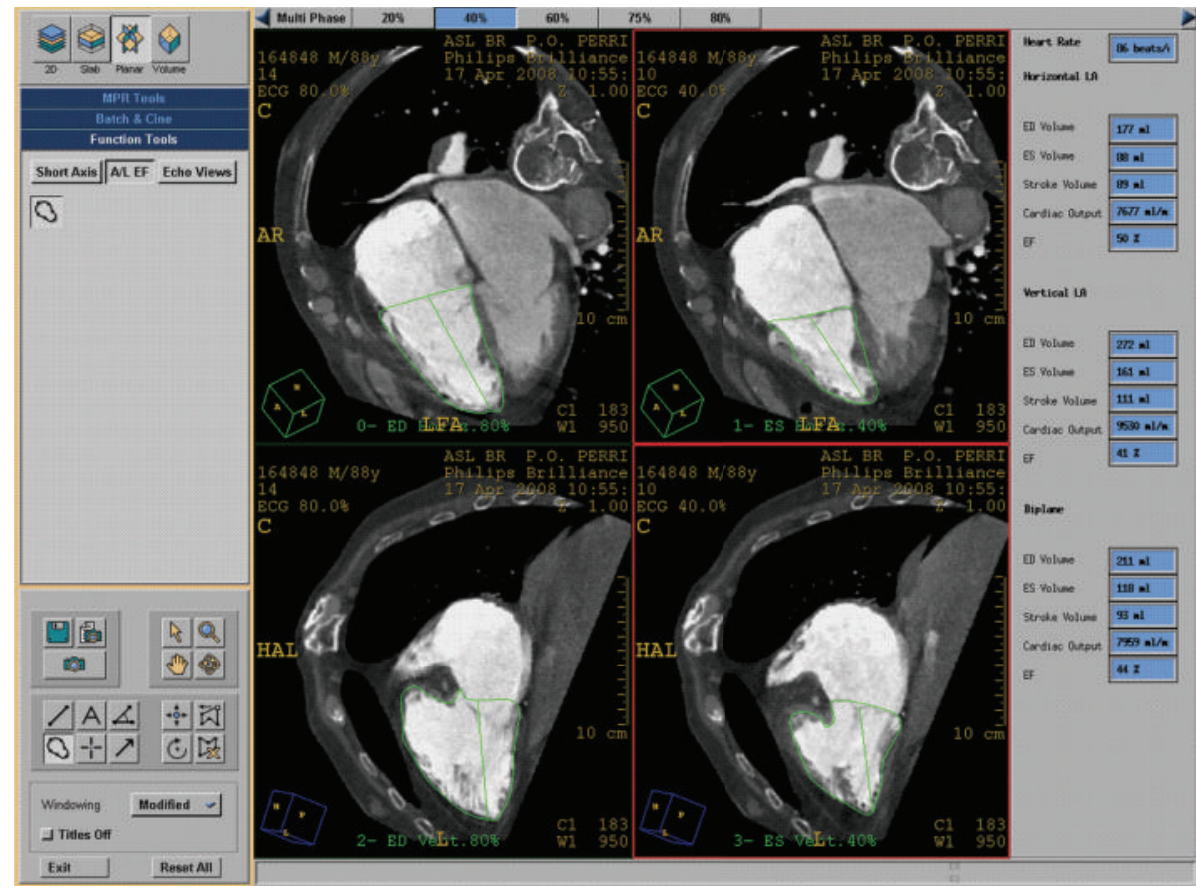

Figure 2: Assessment of cardiac performance. Delimitation of region of interest in end-systolic and end-diastolic phases. Computer-aided calculation of systolic volume and cardiacoutput.

were compared by the analysis of the mean with Student's $t$ test for unpaired data (Tables 1 and 2) by means of computeraided software (Epistat, USA, and Graphpad Prism, USA). The relationships between pulmonary artery features and hemodynamic variables and between cardiac output assessed by MDCT and by Fick method were assessed by linear fitting and least square method.

The study was approved by the Ethics Committee of "Ninetto Melli" Hospital. A written informed consent was obtained from each patient. 


\section{Results}

3.1. Population Studied. Patients showed marked airway obstruction and marked dyspnoea (Table 1). The degree of airway obstruction was advanced, and most patients belonged to the 3rd stage of the disease in both groups (Table 1). The frequency of patients in each stage of COPD was similar in the two groups. The difference between patients with $\mathrm{PH}$ and without $\mathrm{PH}$ was not significant. Hypoxemic hypercapnic chronic respiratory failure could be observed, without significant difference within patients. Patients affected by COPD and PH showed diminished effort tolerance with a significantly lower $6 \mathrm{MWD}$ and an increased PAP and PVR (Table 1), while cardiac output determined by Fick method ( $Q^{\prime}$ Fick) was similar.

3.2. MDCT-Derived PA Diameters and Pulsatility. MDCT allowed suitable imaging and effective measurement in every patient. Pulmonary artery (Table 2) was significantly increased both in axial and in transversal diameters in patients with $\mathrm{PH}$ with a mean difference of about $14 \mathrm{~mm}$ between COPD and COPD with PH.

The diastolic diameters were about $2 \mathrm{~mm}$ less than the systolic ones. Right PA diameters are about $6 \mathrm{~mm}$ lower than those of the main PA. Left PA branches were slightly narrower than right ones in both series.

Two patients with PH had a PA diameter between $30 \mathrm{~mm}$ and $28 \mathrm{~mm}$, the remaining had a diameter larger than $30 \mathrm{~mm}$, while two patients without $\mathrm{PH}$ showed a PA diameter larger than $30 \mathrm{~mm}$. Assuming a cut-off of $28 \mathrm{~mm}$, a sensitivity of $90 \%$, specificity of $85 \%$, a positive predictive power of $85 \%$, and a negative predictive power of $89 \%$ could be obtained. Cross-sectional area of right and left arteries was about $40 \%$ smaller than PA area with narrower values in the left side. It was significantly increased in patients with $\mathrm{PH}$ (Table 2).

The Puls of PA is about 20\% in the PA and in the left branch of COPD patients; right PA showed increased values $(38 \% \pm 5 \%)$ (Table 2). The Puls was significantly diminished (mean 10\%) in patients with PH both in PA and in branches, mainly in the left side (2\%). It was not possible to choose a threshold for the Puls of the main pulmonary artery to diagnose the presence of $\mathrm{PH}$, because of the large transvariance of the distributions between patients with and without PH. Since the stretching of right PA looks enhanced and the difference looks wider, assuming as a threshold the lower limit of right PA Puls (mean minus two standard deviations), a cut-off of $26 \%$ is associated with a sensitivity of $90 \%$, a specificity of $85 \%$, a positive predictive power of $90 \%$, and a negative predictive power of $85 \%$ could be obtained. Left side was not useful to avoid bias in reading the frames due to very low values.

Aorta diameters were normal in both patient sets (transverse diameter $29 \pm 8 \mathrm{~mm}$, axial diameter $29 \pm 5 \mathrm{~mm}$, and area $2.64 \pm .4 \mathrm{~cm}^{2}$ in patients with $\mathrm{PH}$ and $30 \pm 3 \mathrm{~mm}, 30+3 \mathrm{~mm}$ and $2.86 \pm .5 \mathrm{~cm}^{2}$ in patients without $\mathrm{PH}$, resp.), and pulmonary artery/aorta ratio was significantly increased in patients with $\mathrm{PH}(1.2 \pm .2$ in patients with $\mathrm{PH}$ and $.8 \pm .2$ in patients without $\mathrm{PH}$, resp.).

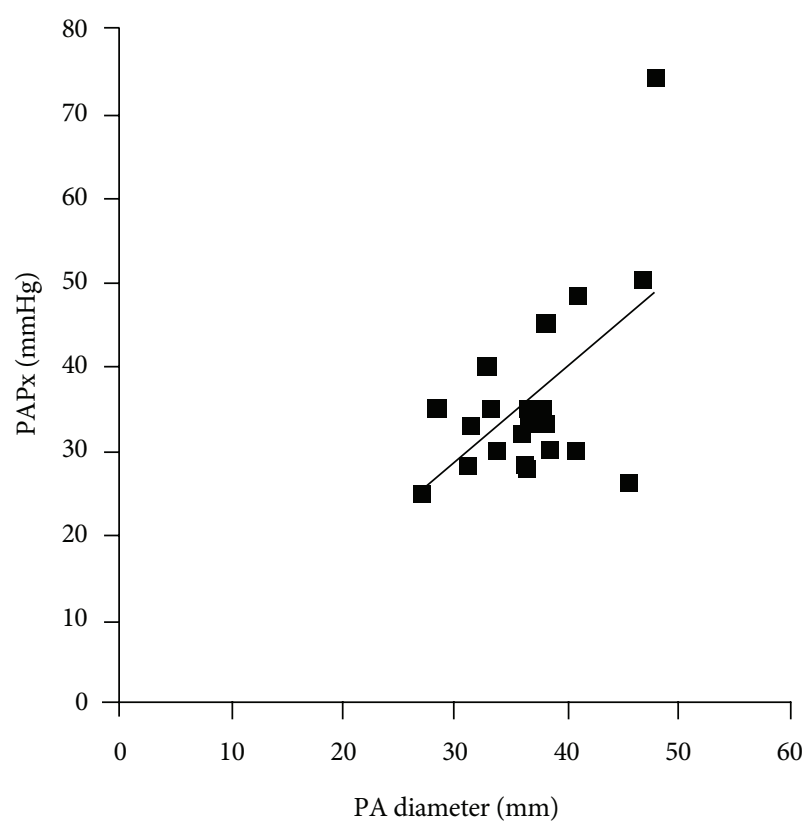

FIGURE 3: Scatter plot of the relationship between pulmonary artery pressure and pulmonary artery Diameter.

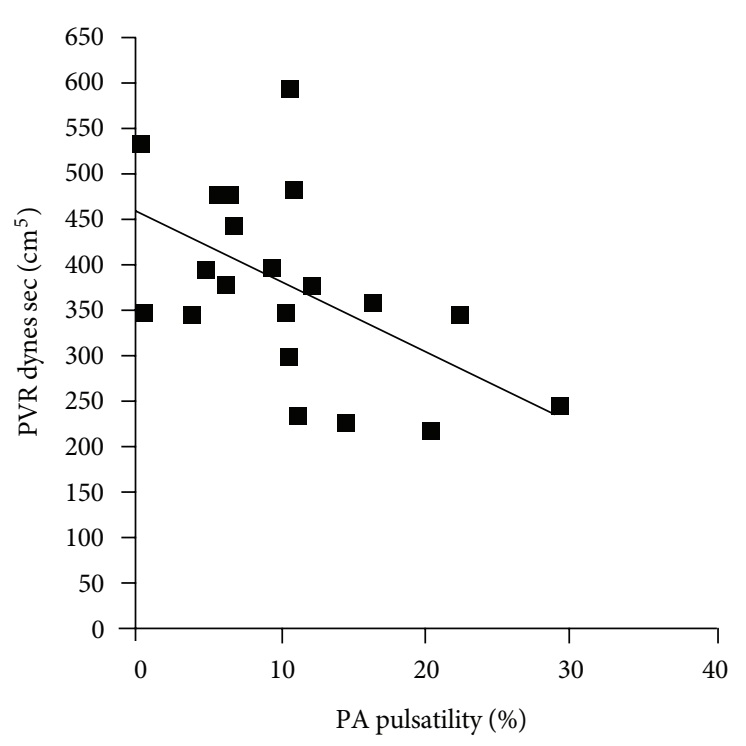

FIGURE 4: The relationship between pulmonary vascular resistance and pulsatility of pulmonary artery.

3.3. The Relationship with Haemodynamics. The Puls was significantly inversely related to PVR (PVR $=458-7.53$ pulsatility $\pm 95 ; r^{2}=.274 ; P=.0177$ ) (Figure 4 ) and PAP $\left(\mathrm{PAP}=38-2.5\right.$ pulsatility $\left.\pm 5, r^{2}=.219 ; P=.0371\right)$ (Figure 5). PA diameter was related to $\mathrm{PH}$ level (Figure 3) (systolic PA diameter $=-6.1+1.14 \mathrm{PAP} \pm .38 ; r^{2}=.33 ; P=$ .0075 ; diastolic PA diameter $=-10+1.32$ PAP; $\pm .34 ; r^{2}=$ $.38 ; P=.0018)$. By grouping of patients according to the WHO staging of $\mathrm{PH}, \mathrm{PA}$ diameter reached an average value of $32 \pm 3 \mathrm{~mm}$ in the 1st stage of $\mathrm{PH}$ (PAP $<25$ at rest but 
TABLE 1: Functional values observed in patients affected by COPD with PH under stable state.

\begin{tabular}{|c|c|c|c|c|}
\hline & Units & $\mathrm{COPD}+\mathrm{PH}$ & COPD & $P$ \\
\hline Age & Years old & $66 \pm 9$ & $65 \pm 10$ & n.s. \\
\hline Height & $\mathrm{cm}$ & $165 \pm 8$ & $167 \pm 10$ & n.s. \\
\hline Weight & kgms & $75 \pm 13$ & $73 \pm 9$ & n.s. \\
\hline GOLD st. II & $\%$ & 25 & 20 & \\
\hline GOLD st. III & $\%$ & 55 & 50 & \\
\hline GOLD st. IV & $\%$ & 20 & 30 & \\
\hline FVC & $\%$ & $49 \pm 18$ & $53 \pm 15$ & n.s. \\
\hline FEV1 & $\%$ & $37 \pm 18$ & $39 \pm 17$ & n.s. \\
\hline $\mathrm{PaO}_{2}$ & $\mathrm{mmHg}$ & $57 \pm 10$ & $58 \pm 9$ & n.s. \\
\hline $\mathrm{PaCO}_{2}$ & $\mathrm{mmHg}$ & $46 \pm 8$ & $46 \pm 9$ & n.s. \\
\hline PAP & $\mathrm{mmHg}$ & $37 \pm 5$ & $18 \pm 5$ & $<.1 \times 10^{-6}$ \\
\hline$Q^{\prime}$ Fick & $\mathrm{L} / \mathrm{m}$ & $4.9 \pm 1.4$ & $5.1 \pm 1.2$ & n.s. \\
\hline$Q^{\prime}$ radiol & $\mathrm{L} / \mathrm{m}$ & $4.1 \pm 1.0$ & $4.2 \pm 1.4$ & n.s. \\
\hline PVR & Dynes $\mathrm{sec}^{-1} \mathrm{~cm}^{-5}$ & $442 \pm 192$ & $120 \pm 70$ & $<.1 \times 10^{-6}$ \\
\hline $6 \mathrm{MWD}$ & Mt & $257 \pm 118$ & $370 \pm 150$ & $<.02246$ \\
\hline Dyspnoea & WHO stage & $\mathrm{III} \pm \mathrm{I}$ & $\mathrm{III} \pm \mathrm{I}$ & n.s. \\
\hline$N$ & $n$ & 20 & 20 & \\
\hline
\end{tabular}

FVC: forced vital capacity as percentage of predicted values; FEV1: forced expired volume in the 1 second as percentage of predicted values; $\mathrm{PaO}_{2}$ : arterial oxygen tension; $\mathrm{PaCO}_{2}$ : arterial carbon dioxide tension; PAP: mean pulmonary artery pressure; $\mathrm{Q}^{\prime}$ : cardiac output measured by radiologic method (radiol) and Fick method (Fick); PVR: pulmonary vascular resistance; $6 \mathrm{MWD}$ : six minutes walking distance; WHO: dyspnoea rating according to WHO stages; and GOLD st. II-III and IV: \% of patients belonging to GOLD stages II, III, and IV.; Values are expressed as mean \pm standard deviation; N: number of observations.

TABLE 2: Pulmonary artery features in COPD.

\begin{tabular}{|c|c|c|c|c|}
\hline & $\mathrm{COPD}+\mathrm{PH}$ & COPD & Units & $P$ \\
\hline \multicolumn{5}{|l|}{ Main pulmonary artery } \\
\hline Transverse systolic $d$ & $36 \pm 5$ & $22 \pm 3$ & $\mathrm{Mm}$ & $<.1 \times 10^{-6}$ \\
\hline Axial systolic $d$ & $38 \pm 4$ & $25 \pm 5$ & $\mathrm{Mm}$ & $<.1 \times 10^{-6}$ \\
\hline Systolic Area & $10.8 \pm 2$ & $4.3 \pm 1$ & $\mathrm{~cm}^{2}$ & $<.1 \times 10^{-6}$ \\
\hline Transverse diastolic $d$ & $34 \pm 2$ & $20 \pm 5$ & $\mathrm{~mm}$ & $<.1 \times 10^{-6}$ \\
\hline Axial diastolic $d$ & $36 \pm 3$ & $22 \pm 4$ & $\mathrm{~mm}$ & $<.1 \times 10^{-6}$ \\
\hline Diastolic area & $9.6 \pm 1$ & $3.4 \pm 1$ & $\mathrm{~cm}^{2}$ & $<.1 \times 10^{-6}$ \\
\hline Pulsatility & $10 \pm 05$ & $21 \pm 07$ & $\%$ & $<.1 \times 10^{-6}$ \\
\hline \multicolumn{5}{|l|}{ Right pulmonary artery } \\
\hline Transverse Systolic $d$ & $30 \pm 1$ & $21 \pm 8$ & $\mathrm{~mm}$ & $<.1 \times 10^{-6}$ \\
\hline Axial Systolic $d$. & $30 \pm 1$ & $21 \pm 7$ & $\mathrm{~mm}$ & $<.1 \times 10^{-6}$ \\
\hline Systolic area & $7.1 \pm 1$ & $3.6 \pm .8$ & $\mathrm{~cm}^{2}$ & $<.1 \times 10^{-6}$ \\
\hline Transverse diastolic $d$ & $28 \pm 2$ & $17 \pm 6$ & $\mathrm{~mm}$ & $<.1 \times 10^{-6}$ \\
\hline Axial diastolic $d$ & $29 \pm 2$ & $16 \pm 6$ & $\mathrm{~mm}$ & $<.1 \times 10^{-6}$ \\
\hline Diastolic area & $6.4 \pm .9$ & $2.2 \pm .6$ & $\mathrm{~cm}^{2}$ & $<.1 \times 10^{-6}$ \\
\hline Pulsatility & $10 \pm 05$ & $38 \pm 05$ & $\%$ & $<.1 \times 10^{-6}$ \\
\hline \multicolumn{5}{|l|}{ Left pulmonary artery } \\
\hline Transverse Systolic $d$ & $28 \pm 2$ & $18 \pm 5$ & $\mathrm{~mm}$ & $<.1 \times 10^{-6}$ \\
\hline Axial Systolic $d$ & $29 \pm 1$ & $19 \pm 4$ & $\mathrm{~mm}$ & $<.1 \times 10^{-6}$ \\
\hline Systolic area & $6.3 \pm .9$ & $2.7 \pm .6$ & $\mathrm{~cm}^{2}$ & $<.1 \times 10^{-6}$ \\
\hline Transverse diastolic $d$ & $28 \pm 2$ & $16 \pm 3$ & $\mathrm{~mm}$ & $<.1 \times 10^{-6}$ \\
\hline Axial diastolic $d$ & $28 \pm 2$ & $19 \pm 5$ & $\mathrm{~mm}$ & $<.1 \times 10^{-6}$ \\
\hline Diastolic area & $6.3 \pm .8$ & $2.1 \pm .5$ & $\mathrm{~cm}^{2}$ & $<.1 \times 10^{-6}$ \\
\hline Pulsatility & $2.1 \pm .5$ & $21 \pm .5$ & $\%$ & $<.1 \times 10^{-6}$ \\
\hline
\end{tabular}

COPD + PH: pulmonary artery diameter in patients with COPD and pulmonary hypertension; COPD: pulmonary artery diameter in patients with COPD and normal pulmonary artery pressure; transverse systolic $d$ : transverse systolic diameter; axial systolic $d$ : axial systolic diameter; and systolic area: sectional systolic area.

Mean \pm SD; $P$ : level of probability, n.s.: not significant; right: right pulmonary artery; left: left pulmonary artery; pulsatility: ratio between systolic and diastolic cross-sectional areas. Number of observations: 20 for each set. 


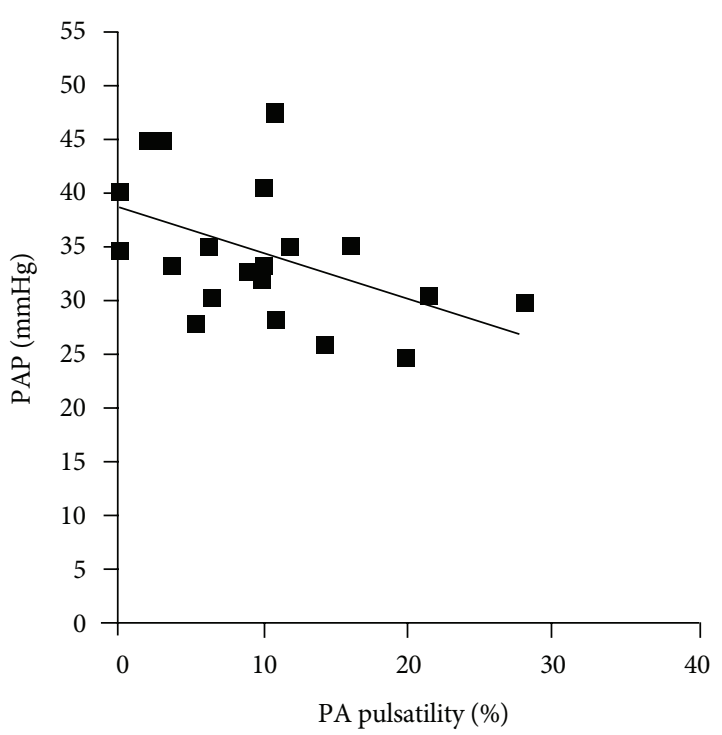

FIGURE 5: The relationship between pulmonary artery pressure and pulsatility of pulmonary artery pressure.

$>25 \mathrm{mmHg}$ on effort), $34 \pm 3 \mathrm{~mm}$ in the 2 nd one (PAP $>25$ and $<35 \mathrm{mmHg}$ ), $36 \pm 3 \mathrm{~mm}$ in the $3 \mathrm{rd}$ one (PAP $>35$ and $<45$ ), and up to $46 \pm 3 \mathrm{~mm}$ in the 4 th stage (PAP $>45 \mathrm{mmHg}$ ). The cardiac output measured by $C T\left(Q^{\prime} \operatorname{rad}=4.1 \pm 1 \mathrm{~L} / \mathrm{m}\right)$ underscores the blood flow measured by Fick method $\left(Q^{\prime}\right.$ Fick). The two measurements are significantly related $\left(Q^{\prime}\right.$ Fick $\left.=1.4+.96 Q^{\prime} \operatorname{rad}(+.09) ; P<.001\right)$.

3.4. Additional Outcomes. CT allowed the assessment of pulmonary nodules (2 patients), arteriovenous malformations (one patient), and severe coronary pathology (three cases). The average absorbed dose was $8 \pm 2 \mathrm{mSv}$.

\section{Discussion}

Our study indicates for the first time the chance to apply CT cardiac imaging software in the study of pulmonary vessels and right ventricular performance. This method allows to appreciate significant differences of PA dimensions, crosssectional areas, and PA stretching in patients with and without $\mathrm{PH}$. The measurements are related to physiological measurements obtained under RHC.

The main limitation of the current study is the low number of observations, due to the low prevalence of the disease and to the difficulties in the recruitment because of the lack of compliance of patients; thus the current data can be regarded just as preliminary outcomes to be confirmed by multicentric studies.

The first outcome is that, according to our results, MDCT cardiac software imaging technique is able to measure the features of pulmonary arteries as well as the right ventricular performance, and it can be used in every COPD patient, while US applicability is limited by the reduced acoustic window and MRI is still not diffuse everywhere and is more expensive and time consuming. MDCT allows as well an effective imaging of the associated pulmonary such as coronary diseases and pulmonary emboli. The applicability is not fully extensible in other pulmonary diseases associated with $\mathrm{PH}$ such as interstitial lung disease, because of the interference of fibrosis upon reading of frames and differences in involvement of vessels $[34,35]$.

The second observation is the observation of a diminished arterial Puls in patients with $\mathrm{PH}$. The applicability in the early diagnosis of $\mathrm{PH}$ looks limited, because the Puls of main PA $(21 \% \pm 07 \%$ in COPD versus $10 \% \pm 05 \%$ in COPD + $\mathrm{PH}$ ) cannot play a role because of the large overlap of the distributions between patients with COPD and COPD with $\mathrm{PH}$, although the pulsatility or right $\mathrm{PA}(38 \pm 05$ in COPD versus $10 \% \pm 05 \%$ in $\mathrm{COPD}+\mathrm{PH}$ ) looks as a promising index to be verified in more extensive studies.

MDCT-derived pulsatility fairly agrees with that measured by IVU and MRI [11-21], indicating a mean pulsatility of $20 \% \pm 5 \%$. The pulsatility significantly diminishes in patients affected by $\mathrm{PH}$, because of arterial remodelling leading to arterial stiffness $[16,17]$.

The relationship between the pulsatility of central arteries and PVR, mainly determined by peripheral vessels, can be explained observing that pathology determines contemporary structural changes in both central and peripheral vessels. The inverse relationship between Puls and PAP depends on the fact that as peripheral vessel's pathology worsens, determining an increase in PVR and PAP, the pulmonary artery dilates according to its elastic modulus and becomes proportionally stiffer with the enlargement of diameters together with the lack of progression of elastic waves into the peripheral units. Furthermore, as the PAP and the right ventricular afterload (determined by the vascular impedance) increase, the right ventricular performance is impaired with the decrease in the systolic volume (the main determinant of pulsatility) and the evidence of pulmonary valve regurgitation [36-38].

The reported value of pulsatility in the prognosis [9] can be explained by the observed relationship between Puls and haemodynamics: the pulmonary artery stretching is dependent on the degree of $\mathrm{PH}$, but it determines as well the ventricular/arterial coupling. Controversial reports indicate higher values in patients responders to vasodilators, suggesting a possible role in the evaluation of reversibility of $\mathrm{PH}$ and the selection of patients responders to calcium channel blockers $[39,40]$.

MDCT allows as well to study the additional features of pulmonary arteries, such as the diameters. According to preliminary reports in the literature [24-29] and our results, the diameters allow the detection of $\mathrm{PH}$ with fairly good sensitivity and specificity. Assuming $28 \mathrm{~mm}$ as the upper limit of normality (mean +2 SD) of PA, a sensitivity of $90 \%$, a specificity of $85 \%$, and a positive predictive value of $96 \%$ can be obtained by our study with outcomes similar to those obtained in larger series [24-29]. The variance observed around the slope of the relationship between diameters and PAP can be explained by several reasons: (1) differences in the amount of elastic bundles of the main pulmonary artery tissues; (2) dilation determined by previous loads, such as higher PAP peaks preceding the measures, heavier than 
the actual measured PAP; (3) differences in cardiac output; (4) different body surface area, since cross-sectional area of PA and left branch corrected by body surface area were significantly related to PAP; and (5) time of measurement referred to the natural history of disease, since pulmonary artery progressively increases even in case of reduction of PVR by vasodilators, due to intrinsic vessel properties independent of pressure and flow changes. Other relevant findings in the literature are the arterial/bronchial ratio $>1,1$ in lobar arteries and the ratio PA/Aorta higher than 1 and close to $1.2 \pm .3$, as found in our patients as well [24-29].

In conclusion, MDCT could be applied in every COPD patient, allowing the measurement of PA pulsatility, lesser in patients affected by $\mathrm{PH}$ and closely related to the haemodynamic variables. Pulsatility can contribute to raise the suspicion of the existence of $\mathrm{PH}$ together with the measurement of diameters. MDCT allows as well an insight in to the performance of RV by means of the assessment of the cardiac output related to the hemodynamic measures, and it offers the chance to verify additional thoracic or cardiac lesions, justifying the radiation dose absorbed. These findings are relevant not only as scientific outcomes but as well in clinical practice because they offer a suitable and also available technique to be applied once in primary health care when the suspect of $\mathrm{PH}$ is raised.

\section{Summary}

The aim was to assess if multidetector computed tomography (MDCT) is able to measure pulmonary artery (PA) pulsatility (Puls) in patients affected by chronic obstructive pulmonary disease (COPD) and to ascertain whether Puls is different in patients with and without pulmonary hypertension $(\mathrm{PH})$ and whether it is related to haemodynamics. Statistical analysis by Student's $t$ test allowed the comparison of data between patients with and without $\mathrm{PH}$. The linear fitting and the least square method allowed to assess a significant relationship between haemodynamics and imaging. MDCT allowed the imaging and the measurement of PA features in all the patients. We selected two groups of COPD patients, the first one with $\mathrm{PH}$, and the second one without $\mathrm{PH}$. In patients with COPD and PH PAP and PVR were increased (PAP $37 \pm 18$ versus $18 \pm 5 \mathrm{mmHg}$, PVR $442 \pm 192$ versus $120 \pm 70$ dynes $\mathrm{s}^{-1} \mathrm{~cm}^{-5}$ ) and PA showed increased diameters (transverse $36 \pm 5 \mathrm{~mm}$, axial $38 \pm 4 \mathrm{~mm}$ versus $22 \pm 3$ and $25 \pm 5$, resp.), increased cross-sectional area (10 \pm 08 versus $\left.4 \pm 1 \mathrm{~cm}^{2}\right)$, and reduced pulsatility $(21 \pm 7$ versus $10 \pm 5 \%)$. Arterial stretching was decreased in patients with $\mathrm{PH}$ $(10 \% \pm 5$ versus $21 \pm 7 \%$ in non- $\mathrm{PH})$ and significantly related to pulmonary vascular resistances (PVRs) (PVR $=458-7.53$ pulsatility $\left.\pm 95 ; r^{2}=.274 ; P=.0177\right)$ and pulmonary artery pressure (PAP) $\mathrm{PAP}=38-2.5$ pulsatility $\pm 5, r^{2}=$ $.219 ; P=.0371)$. Cardiac output measured by MDCT was significantly related to that obtained by Fick method (4.1 \pm $1.0 \mathrm{~L} / \mathrm{m}$ versus $4.9 \pm 1.4$, resp.) and was not different in the two groups. The diameters of PA allow to identify patients with $\mathrm{PH}$ with a confident specificity and sensitivity: assuming a cut-off of $28 \mathrm{~mm}$ for the diameter of PA, it was possible to diagnose pulmonary hypertension with a sensitivity of $90 \%$, a specificity of $85 \%$, a positive predictive power of $85 \%$, and a negative predictive power of $89 \%$. While the distribution of PA Puls was overlapping, the Puls of right PA, assuming a cut off of $26 \%$, allowed to diagnose $\mathrm{PH}$ with a specificity of $85 \%$, a positive predictive power of $90 \%$, and a negative predictive power of $85 \%$. These preliminary observations, to be confirmed in multicentric and more extensive studies, indicate $\mathrm{MDCT}$ as a suitable technique in COPD, able to measure the Puls and the dimensions of pulmonary arteries as well as the right ventricular functional parameters.

\section{Abbreviations}

$\begin{array}{ll}\text { Puls: } & \text { Pulsatility } \\ \text { MDCT: } & \text { Multidetector tomography } \\ \text { PH: } & \text { Pulmonary hypertension } \\ \text { PAP: } & \text { Pulmonary artery pressure } \\ \text { PA: } & \text { Pulmonary artery } \\ \text { PVRs: } & \text { Pulmonary vascular resistances } \\ Q^{\prime}: & \text { Cardiac output }\end{array}$

COPD: Chronic obstructive pulmonary disease

RHC: Right heart catheterization.

\section{Conflict of Interest}

The authors declare that they have no conflict of interests, and the study was supported by no grant.

\section{References}

[1] G. Simonneau, N. Galie', and L. Rubin, "Clinical classification of pulmonary arterial hypertension," Journal of the American College of Cardiology, vol. 43, no. 12, supplement, pp. s5-s12, 2004.

[2] R. Kessler, M. Faller, E. Weitzenblum et al., “"Natural history” of pulmonary hypertension in a series of 131 patients with chronic obstructive lung disease," American Journal of Respiratory and Critical Care Medicine, vol. 164, no. 2, pp. 219-224, 2001.

[3] R. Naejie and A. Barbera, "Pulmonary hypertension associated with COPD," Critical Care, vol. 5, pp. 286-291, 2001.

[4] O. Firă-Mladinescu, L. Vasile, C. Suciu et al., "Some aspects of pulmonary vascular remodelling in smokers and patients with mild COPD," Pneumologia, vol. 57, no. 1, pp. 7-16, 2008.

[5] S. Santos, V. I. Peinado, J. Ramirez et al., "Chracterization of pulmonary vascular remodelling in smokers," European Respiratory Journal, vol. 19, no. 4, pp. 632-638, 2002.

[6] K. W. Kang, H. J. Chang, Y. J. Kim, B. W. Choi et al., "Cardiac magnetic resonance imaging-derived pulmonary artery distensibility index correlates with pulmonary artery stiffness and predicts functional capacity in patients with pulmonary arterial hypertension," Circulation Journal, vol. 75, no. 9, pp. 2244-2251, 2011.

[7] Y. Fukumoto, "Pulmonary artery distensibility index is a noninvasive useful marker of pulmonary arterial hypertension progression," Circulation Journal, vol. 75, no. 9, pp. 2058-2059, 2011.

[8] A. J. Swift, S. Rajaram, R. Condliffe, D. Capener et al., "Pulmonary artery relative area change detects mild elevations in pulmonary vascular resistance and predicts adverse outcome 
in pulmonary hypertension," Investigative Radiology, vol. 47, no. 10, pp. 571-577, 2012.

[9] C. T. J. Gan, J. W. Lankhaar, N. Westerhof et al., "Noninvasively assessed pulmonary artery stiffness predicts mortality in pulmonary arterial hypertension," Chest, vol. 132, no. 6, pp. 19061912, 2007.

[10] J. Rodés-Cabau, E. Domingo, A. Román et al., "Intravascular ultrasound of the elastic pulmonary arteries: a new approach for the evaluation of primary pulmonary hypertension," Heart, vol. 89, no. 3, pp. 311-315, 2003.

[11] A. C. Borges, R. Wensel, C. Opitz, U. Bauer, G. Baumann, and F. X. Kleber, "Relationship between haemodynamics and morphology in pulmonary hypertension. A quantitative intravascular ultrasound study," European Heart Journal, vol. 18, no. 12, pp. 1988-1994, 1997.

[12] C. E. Weinberg and J. R. Hertzberg, "Use of intravascular ultrasound to measure local compliance of the pediatric pulmonary artery: in vitro studies," Journal of the American Society of Echocardiography, vol. 15, no. 12, pp. 1507-1514, 2002.

[13] E. Domingo, R. Aguilar, M. López-Meseguer, G. Teixidó, M. Vazquez, and A. Roman, "New concepts in the invasive and non invasive evaluation of remodelling of the right ventricle and pulmonary vasculature in pulmonary arterial hypertension," Open Respiratory Medicine Journal, vol. 3, pp. 31-37, 2009.

[14] E. M. Lau, N. Iyer, R. Ilsar, B. P. Bailey, M. R. Adams, and D. S. Celermajer, "Abnormal pulmonary artery stiffness in pulmonary arterial hypertension: in vivo study with intravascular ultrasound," PLoS ONE, vol. 7, no. 3, Article ID e33331, 2012.

[15] M. R. Fisher, G. J. Criner, A. P. Fishman et al., "Estimating pulmonary artery pressures by echocardiography in patients with emphysema," European Respiratory Journal, vol. 30, no. 5, pp. 914-921, 2007.

[16] S. Ghio, A. S. Pazzano, C. Klersy et al., "Clinical and prognostic relevance of echocardiographic evaluation of right ventricular geometry in patients with idiopathic pulmonary arterial hypertension," American Journal of Cardiology, vol. 107, no. 4, pp. 628632, 2011.

[17] C. Ginghina, D. Muraru, A. Vladaia et al., "Doppler flow patterns in the evaluation of pulmonary hypertension," Romanian Journal of Internal Medicine, vol. 47, no. 2, pp. 109-121, 2009.

[18] A. Vonk-Noordegraaf, J. T. Marcus, S. Holverda, B. Roseboom, and P. E. Postmus, "Early changes of cardiac structure and function in COPD patients with mild hypoxemia," Chest, vol. 127, no. 6, pp. 1898-1903, 2005.

[19] R. W. W. Biederman, "Cardiovascular magnetic resonance imaging as applied to patients with pulmonary arterial hypertension," International Journal of Clinical Practice, vol. 63, no. 162, pp. 20-35, 2009.

[20] N. Creuzé, S. Hoette, and D. Chemla, "Magnetic resonance imaging and pulmonary hypertension: towards an improved evaluation of right heart and pulmonary circulation," Presse Medicale, vol. 40, supplement 1, pp. 1S21-1S27, 2011.

[21] C. Jardim, C. E. Rochitte, M. Humbert et al., "Pulmonary artery distensibility in pulmonary arterial hypertension: an MRI pilot study," European Respiratory Journal, vol. 29, no. 3, pp. 476-481, 2007.

[22] J. B. A. Haimovici, B. Trotman-Dickenson, E. F. Halpern et al., "Relationship between pulmonary artery diameter at computed tomography and pulmonary artery pressures at right-sided heart catheterization," Academic Radiology, vol. 4, no. 5, pp. 327334, 1997.
[23] E. Abel, A. Jankowski, C. Pison, J. L. Bosson, H. Bouvaist, and G. R. Ferretti, "Pulmonary artery and right ventricle assessment in pulmonary hypertension: correlation between functional parameters of ECG-gated CT and right-side heart catheterization," Acta Radiologica, vol. 53, no. 7, pp. 720-727, 2012.

[24] B. Boerrigter, G. J. Mauritz, J. T. Marcus et al., "Progressive dilatation of the main pulmonary artery is a characteristic of pulmonary arterial hypertension and is not related to changes in pressure," Chest, vol. 138, no. 6, pp. 1395-1401, 2010.

[25] R. Condliffe, M. Radon, J. Hurdman et al., "CT pulmonary angiography combined with echocardiography in suspected systemic sclerosis-associated pulmonary arterial hypertension," Rheumatology, vol. 50, no. 8, pp. 1480-1486, 2011.

[26] R. S. Dusaj, K. C. Michelis, M. Terek et al., "Estimation of right atrial and ventricular hemodynamics by CT coronary angiography," Journal of Cardiovascular Computed Tomography, vol. 5, no. 1, pp. 44-49, 2011.

[27] F. Y. Lin, R. B. Devereux, M. J. Roman et al., “The right sided great vessels by cardiac multidetector computed tomography. Normative reference values among healthy adults free of cardiopulmonary disease, hypertension, and obesity," Academic Radiology, vol. 16, no. 8, pp. 981-987, 2009.

[28] R. T. Tan, R. Kuzo, L. R. Goodman, R. Siegel, G. B. Haasler, and K. W. Presberg, "Utility of CT scan evaluation for predicting pulmonary hypertension in patients with parenchymal lung disease," Chest, vol. 113, no. 5, pp. 1250-1256, 1998.

[29] R. A. Pauwels, A. S. Buist, P. M. A. Calverley, C. R. Jenkins, and S. S. Hurd, "Global strategy for the diagnosis, management, and prevention of chronic obstructive pulmonary disease: NHLBI/WHO Global Initiative for Chronic Obstructive Lung Disease (GOLD) workshop summary," American Journal of Respiratory and Critical Care Medicine, vol. 163, no. 5, pp. 12561276, 2001.

[30] P. Quanjer, "ERS official statement on lung volumes and expiratory flows," European Respiratory Journal, vol. 6, supplement 16, pp. 5-40, 1993.

[31] R. O. Crapo, R. Casaburi, A. L. Coates et al., "ATS statement: guidelines for the six-minute walk test," American Journal of Respiratory and Critical Care Medicine, vol. 166, no. 1, pp. 111117, 2002.

[32] D. A. Mahler and A. Harvey, Dyspnea in Fishman AP Pulmonary Rehabilitation, Marcel Dekker, New York, NY, USA, 1996.

[33] N. Buchbinder and W. Ganz, "Hemodynamic monitoring: invasive techniques," Anesthesiology, vol. 45, no. 2, pp. 146-155, 1976.

[34] D. A. Zisman, A. S. Karlamangla, D. J. Ross et al., "Highresolution chest CT findings do not predict the presence of pulmonary hypertension in advanced idiopathic pulmonary fibrosis," Chest, vol. 132, no. 3, pp. 773-779, 2007.

[35] E. H. Alhamad, A. A. Al-Boukai, F. A. Al-Kassimi et al., "Prediction of pulmonary hypertension in patients with or without interstitial lung disease: reliability of CT findings," Radiology, vol. 260, pp. 875-883, 2011.

[36] M. Remy-Jardin, D. Delhaye, A. Teisseire, C. Hossein-Foucher, A. Duhamel, and J. Remy, "MDCT of right ventricular function: impact of methodologic approach in estimation of right ventricular ejection fraction," American Journal of Roentgenology, vol. 187, no. 6, pp. 1605-1609, 2006.

[37] P. J. Kilner, R. Balossino, G. Dubini et al., "Pulmonary regurgitation: the effects of varying pulmonary artery compliance, and 
of increased resistance proximal or distal to the compliance," International Journal of Cardiology, vol. 133, no. 2, pp. 157-166, 2009.

[38] G. R. Stevens and A. Garcia-Alvarez, "RV dysfunction in pulmonary hypertension is independently related to pulmonary artery stiffness," JACC: Cardiovascular Imaging, vol. 5, no. 4, pp. 378-387, 2012.

[39] D. D. Ivy, S. R. Neish, O. A. Knudson et al., "Intravascular ultrasonic characteristics and vasoreactivity of the pulmonary vasculature in children with pulmonary hypertension," American Journal of Cardiology, vol. 81, no. 6, pp. 740-748, 1998.

[40] J. C. Grignola, E. Domingo, R. Aguilar et al., "Acute absolute vasodilatation is associated with a lower vascular wall stiffness in pulmonary arterial hypertension," International Journal of Cardiology, 2011. 


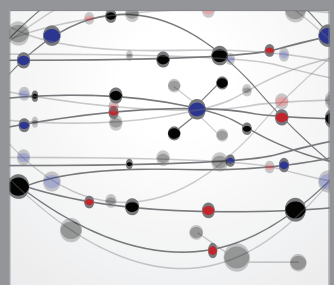

The Scientific World Journal
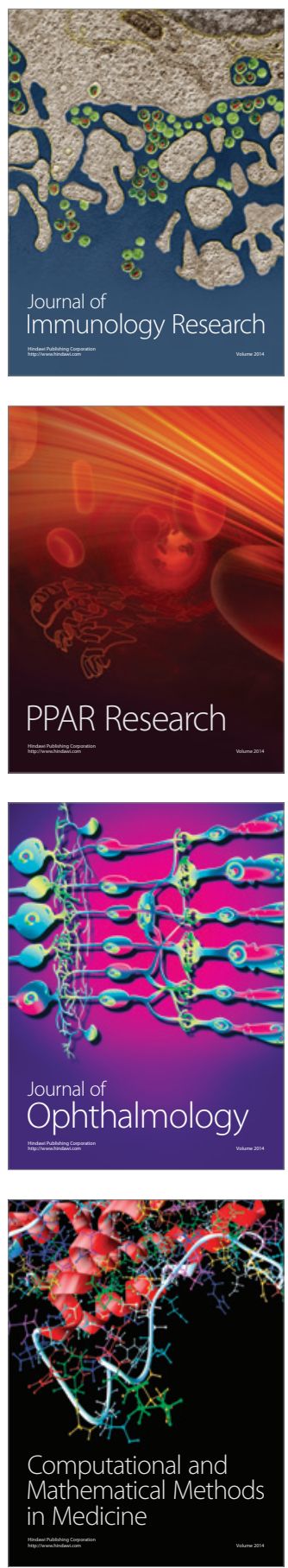

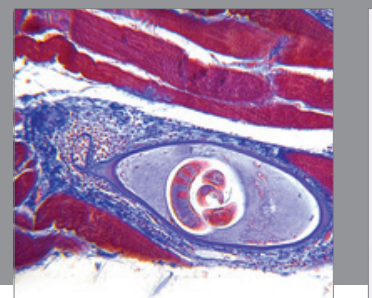

Gastroenterology

Research and Practice
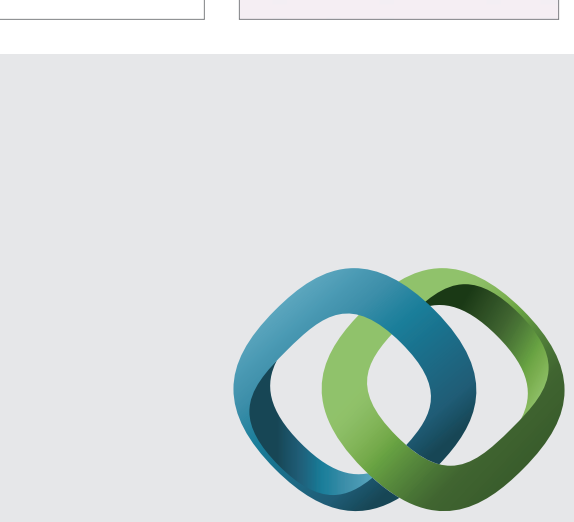

\section{Hindawi}

Submit your manuscripts at

http://www.hindawi.com
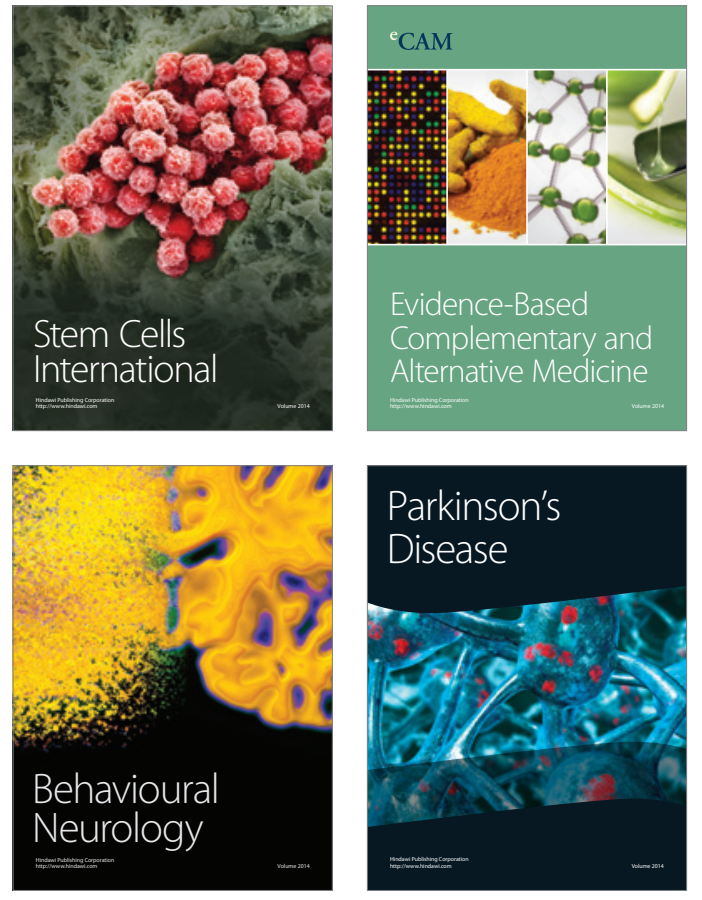
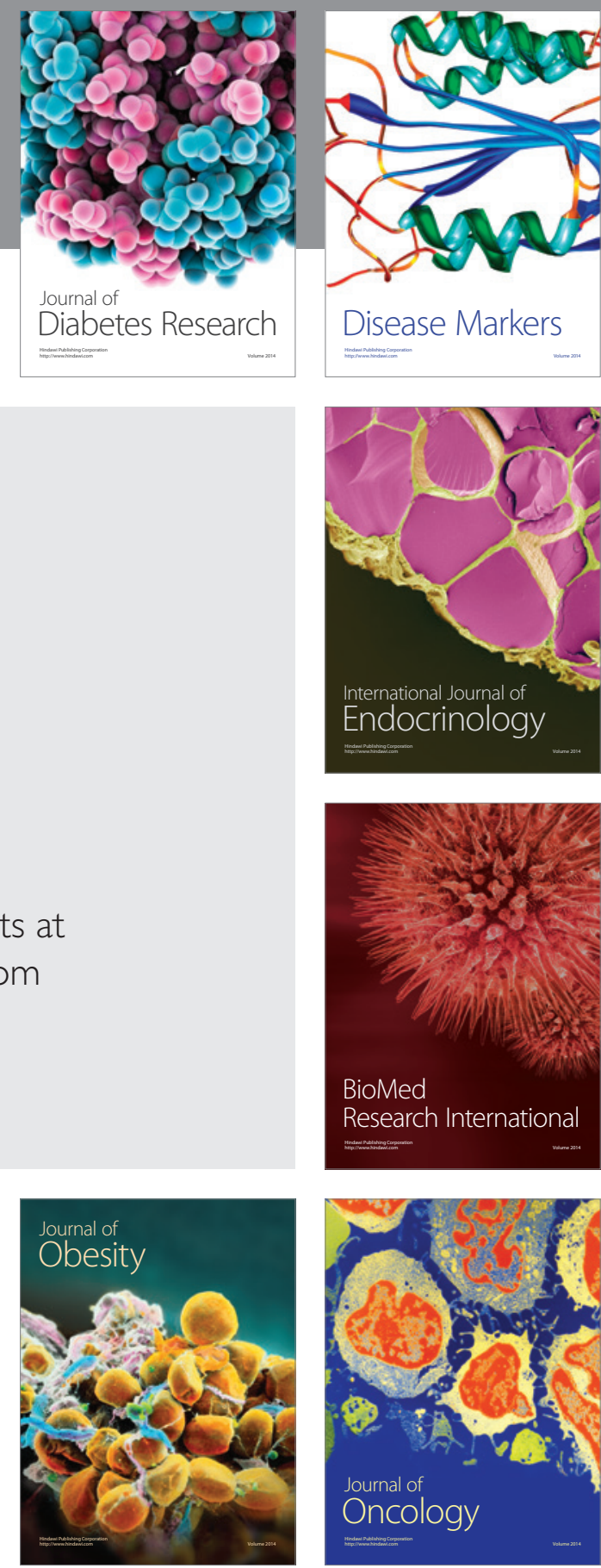

Disease Markers
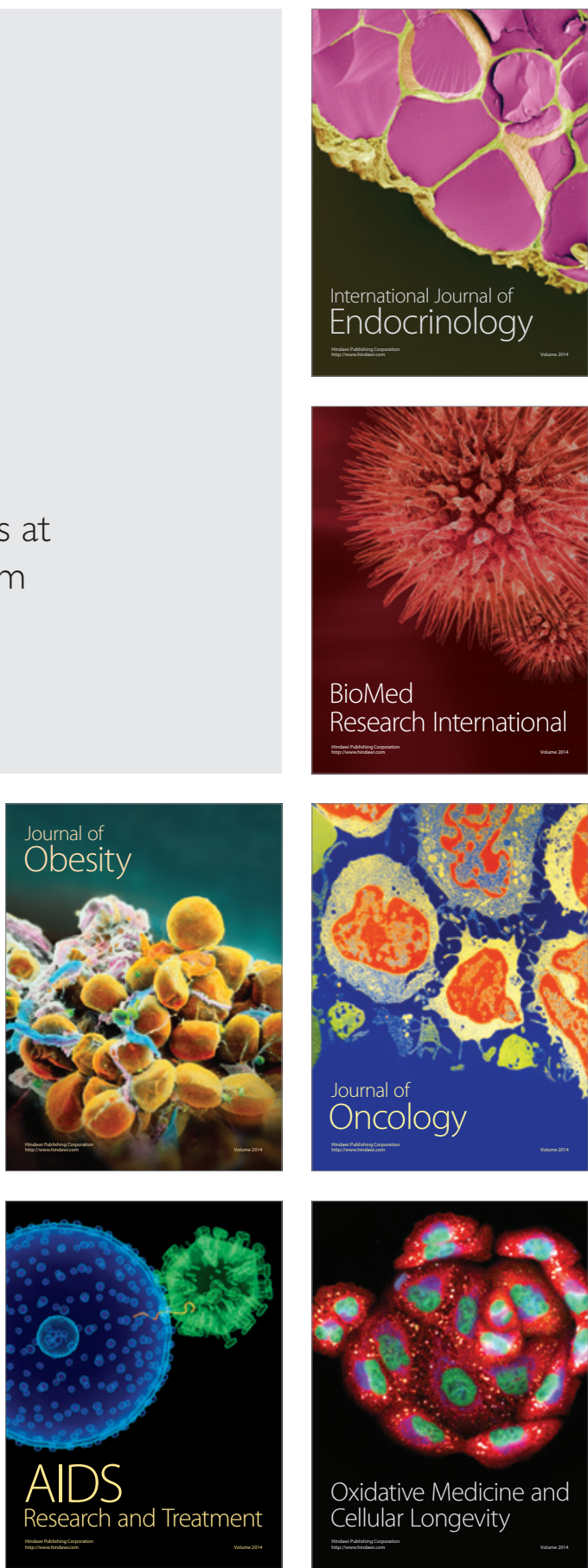